\title{
Visionizing a Fiat Cryptocurrency
}

\author{
Sand Salhout \\ Dual Studies Business Administration Program, Faculty of Business and Economics \\ Al-Quds University, Abu Dis, East Jerusalem \\ E-mail: ssalhout@staff.alquds.edu
}

Assoc. Prof. Dr. Clemens Bechter (Corresponding author)

Thammasat Business School, The Prachan, Bangkok 10200, Thailand

E-mail: clemensbechter@tbs.tu.ac.th

Received: July 17, 2018 Accepted: August 23, 2018 Published: September 1, 2018

doi:10.5296/csbm.v5i2.13391 URL: http://dx.doi.org/10.5296/csbm.v5i2.13391

\begin{abstract}
Bitcoin is part of a wider range of opportunities to creating cryptocurrencies based on blockchains. Research has shown that the existing cryptocurrencies are very volatile and often illiquid. A cryptocurrency endorsed by a Central Bank, as the starter of a private blockchain, will most likely show more stability. It may even take the form of legal tender. However, since almost all countries have their own currency already, the governments will not feel the need for an additional currency. One exception is Palestine. It enjoyed the privilege of having its own currency in the past. The purpose of this research is the evaluation of the feasibility of a cryptocurrency as legal tender. The authors recommend a new Palestinian Pound built on a private blockchain using Hyperledger. In practice it would not differ from any other fiat (legal) currency. The current weakness of not having its own currency could be turned into a strength by becoming the most advanced economy where transactions are transparent and efficient. This paper fills a research gap by analyzing the opportunities of a cryptocurrency that is actual legal tender and endorsed by a monetary authority.
\end{abstract}

Keywords: cryptocurrency, private blockchain, public blockchain, cyberocracy, Hyperledger, Ethereum, Palestine Monetary Authority. 


\section{Al Macrothink}

\section{Cryptocurrencies}

As the name suggests, cryptocurrencies are built on cryptography and blockchain technology. Cryptocurrencies are difficult to counterfeit because of this encryption feature and the fact that blockchains are relying on consensus. Most famous are Bitcoin and Ether. Their values fluctuate heavily, see Figure 1.

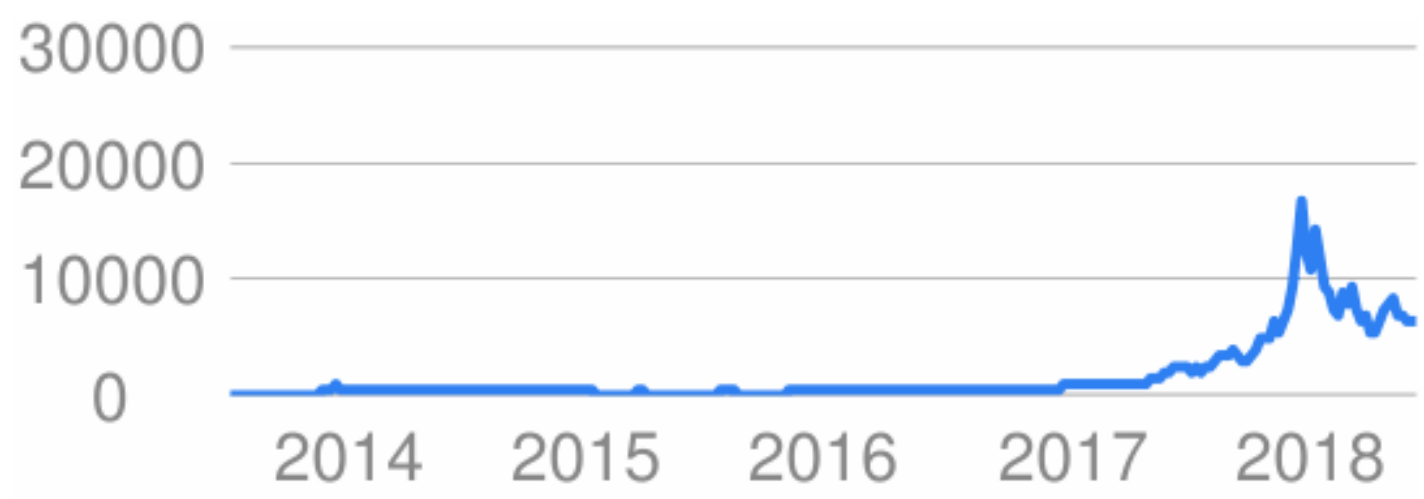

Figure 1. Bitcoin Price in EUR (source: bitcoin.com)

According to Weaver (2018), cryptocurrencies are vulnerable in four aspects, namely, technical risks, economic risks, systemic risks, and societal risks. Energy consumption and associated costs are technical risks. It is estimated that today the Bitcoin mining process consumes as much energy as the whole of Ireland. Cryptocurrencies may need more energy than there is globally available. The economic risk is large scale theft. Since cryptocurrencies use private keys, anyone who gains access to a private key can move the cryptocurrency. The systemic risk is that cryptocurrencies rely on P2P networks and as such can spread computer worms easily. A malicious worm could spread within seconds across large networks. The societal risk is that cryptocurrencies can be used undetectably for criminal activity such as money laundering.

In contrast, Corbet et al. (2018) highlight the benefits. They found that cryptocurrencies do not correlate with traditional asset prices. In times of financial crises, the investment in cryptocurrencies may be a desirable diversification strategy for a portfolio to minimize the risks of a total financial loss. Saeed and Zahid (2016) found that in times of a financial crisis, not only private and corporate investors must fear losses, but also large commercial banks. Cryptocurrencies may prove to be a good hedge for them. Wei (2018) looked at 456 different cryptocurrencies and found that most are illiquid. Whereas the usefulness of Bitcoins and other cryptocurrencies can be disputed, the underlying technology, blockchains, is gaining a foothold in many industries. Davies (2015) brought it to the point by saying "Blockchain is to Bitcoin, what the internet is to email. A big electronic system, on top of which you can build applications. Currency is just one." 


\section{Blockchain and Distributed Ledger Technologies}

A blockchain consists of blocks which are interlinked. Each block contains a hash function which is derived from the previous block. It also includes a timestamp and data about a transaction (e.g., product shipment or payment). A Blockchain relies on a peer-to-peer (P2P) network infrastructure of many computers (nodes). Changes in the chain can only be made if the majority of nodes agree to the change. Blockchains such as Ethereum have the same objectives as distributed leger technologies which is the digitization of goods and assets. Both rely on decentralization in form of distributing data across many computers. A distributed ledger is a database that is shared across a P2P network consisting of multiple nodes/computers. Each member of the network (computer) has its own copy of the ledger. For example, Everledger is a distributed ledger that assures the identity of diamonds, from the mine to the wholesaler to the seller to the owner i.e. it documents and archives the whole supply chain over a large P2P network (Garrett, 2017).

In contrast to traditional organizations which are structured in a top-down manner with many layers of management, the blockchain concept is the exact opposite by being structured bottom-up. Blockchains work with decentralized autonomous organizations (DAOs). DAOs bind people together, not by a formal contract, but by transparent rules (smart contracts) that are written into the software. For example, a smart contract will specify when a payment must be made i.e. under which (fulfilled) conditions the money must be released. No central entity controls blockchains. The smart contract is automatically executed as soon as contract conditions have been met.

Blockchain is a good idea when verifying a chain of events. For example, when buying a sausage, the buyer could trace back from which animal and farmer the meat stems from. And the seller could trace back from where the money comes from that the buyer is paying with. Theoretically it would be possible to store all this information on a server, but single machines can be hacked and information changed. A network of millions of peer-to-peer computers cannot (so the theory but see 'Forking' further down).

There are two types of blockchains: public and private ones. Technically they operate in the same way. The only difference is the community i.e. the participants. A private blockchain works on the principle of 'on invitation only'. One cannot join without being invited. After having accepted the invitation one has the permission of participating in the network and execute the so-called consensus protocol which is an agreement on the validity of data amongst a distributed P2P network. Everyone in the network is maintaining the shared ledger. Every participant in a private blockchain network needs an invitation which comes from the network starter (e.g. an organization) or comes automatically when certain conditions are met. Initially, these joining conditions have been set by the network starter. Private blockchains are often based on Hyperledger Frameworks. Hyperledger Fabric is currently the leading infrastructure on which private blockchains are based.

Berg (2017) compared blockchains with diplomatic protocols and found striking similarities. Both rely on records detailing "past dealings, public and ritualistic verification of transactions, and game-theoretic mechanisms of reciprocity" (Berg, 2017, p.55). Diplomatic protocols 
allowed facilitate peaceful settling of disputes. The same hope is put into blockchains by using smart contracts.

What makes blockchains difficult to understand are the technical terms that insiders use such as 'tokenization' or soft and hard 'forking' etc. Smart contracts are based on an infrastructure called Zeppelin. All this is new territory, even for most IT developers.

\subsection{Ethereum}

One can start a blockchain-based business without having to develop a platform. Today, the token concept based on Ethereum is the backbone of most start-ups and their initial coin offerings (ICOs). Ethereum is a blockchain-based, open-source, public, P2P platform and operating system. The company is incorporated in Zug, Switzerland. It has attracted a lot of attention from businesses and is on its way to overtake Bitcoin's popularity by focusing more on actual business transactions than a single currency. Ether, its virtual currency, is the second largest cryptocurrency by market capitalization, although still only 1/10th of a Bitcoin.

The most widely used token standard for Ethereum is called ERC20. Developers must follow certain criteria, as laid down in ERC20, so blockchains can be shared across the Ethereum world.

There are already browser extensions for Ethereum available such as MetaMask which connect a user with Ethereum applications (called dApps) that can also serve as a digital wallet i.e. one can store digital currencies in it and use them to buy anything that can be bought with Ethers.

In June 2016 the unthinkable happened: A project named 'The DAO' was exploited for 2.6 million Ethers (an Ether is currently around 500 US\$) resulting in a massive transfer of Ethereum's overall money supply to the hands of a malicious individual/group. It was decided that the exploit be reversed with the stolen funds being returned to their rightful owners. But this broke the promise of a blockchain being immutable i.e. cannot be changed. Such change in a chain is called (hard/soft) Forking. The claim that blockchains store digitized truth, distributed across thousands of nodes (computers) in locations across the world (P2P networks) and acting as an immutable ledger which separates it from traditional databases, was proven false. This came as a shock.

One must understand that there are three layers in blockchain technology: the maths/hash function which cannot be manipulated, the code (can be manipulated, see Forking as result), and the community using the technology. Trust is probably the most important part when it comes to the community buying into a system's worthiness. If the reputation management and communication is done well, users will likely have not a problem with Forking and other changes along the way.

Since 2016 there is a running debate that if you don't believe in the immutability of a blockchain then you might as well be running a public database on a server or use distributed ledger technologies. 


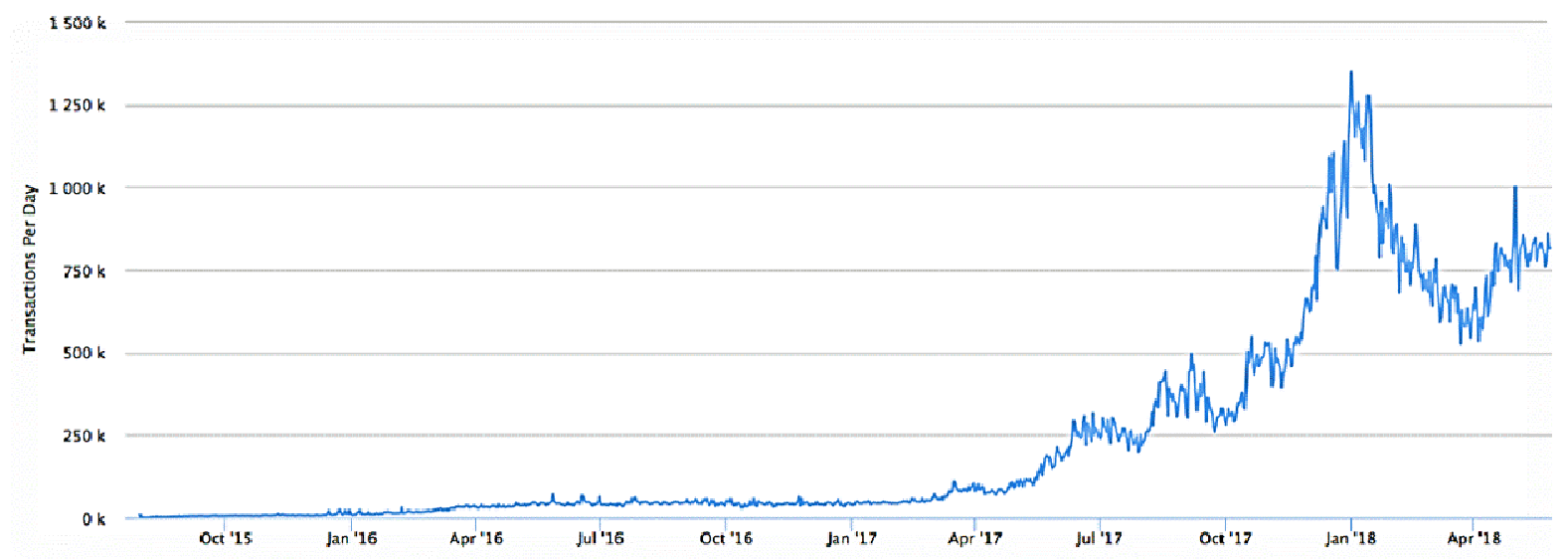

Figure 2. Ethereum Transactions per day (source: etherscan.io)

In January 2018, see Figure 2, the number of transactions on the Ethereum blockchain surpassed 1 million per day. An estimated number of 250,000 developers work worldwide with Ethereum according to etherscan.io. Depending what kind of metrics one applies, its blockchain market share is between 80 and 90 percent.

Like any other technology, Ethereum does not only come with benefits but carries potential risks too. There are two main objections against Ethereum which can also be said about blockchain in general. Firstly, if the whole world goes blockchaining then the data volume will be so huge that it cannot be stored even when considering that the Hash function is just around $15 \mathrm{~kb}$ each. Assuming that the world will not adopt Ethereum at once and not each and every transaction will be run on Ethereum then the storage issue becomes less worrying. The second concern is the energy consumption. Today, the energy costs of mining Bitcoins exceeds the rewards already. Even when considering cloud computing and using cheap energy countries like China this might become a serious problem long-term. Estimated costs of mining one Bitcoin in South Korea is USD 26,000 Dollar, in Germany 14,300 and in Norway USD 7,800 (Lefdal Mine Datacenter located in a former mine). The current value of a Bitcoin is less than that. It is estimated that in 2018 Bitcoin mining will consume 130 terawatt hours which is equivalent to the electricity consumption of the whole of Argentina or Ireland for the same year (Meyer, 2018). On the other hand, Ethereum will make energy markets more efficient (PR, 2017).

\subsection{Applications}

There are public as well as private commercial and non-commercial applications of blockchain technology.

Even governments try to make use of blockchains. At the beginning of 2018 the Brazilian government announced plans to move petitions and public discussions of upcoming laws onto Ethereum (Young, 2018). Canada plans to use Ethereum to ease citizens' concerns of misappropriation of public grants and fight corruption (Nation, 2018). Chile plans to use Ethereum to track data of the energy grid and make the data publicly available (Floyd, 2018). 
Dubai wants to use blockchain for visa applications and license renewals, which account for over 100 million documents each year. The government hopes to save more than 100 metric tons of $\mathrm{CO} 2$ emissions due to a reduction in the number of trips and saving 25 million hours of economic productivity by a reduction in document processing time (SmartDubai, 2018). Estonia plans to become the first digital republic altogether (Heller, 2017).

One of the largest non-commercial implementations of the Ethereum blockchain for a charitable cause is run by the United Nation's World Food Program (Paynter, 2017). It was designed to direct resources to thousands of Syrian refugees in Jordan by giving them cryptocurrency-based vouchers (Ethers) that can be redeemed in participating markets. The system has been successfully running in supermarkets in Jordan's refugee camps since May 2017. Currently, the agency is in talks with partners in humanitarian and private organizations to help achieving the Zero Hunger goal by 2030 by using blockchains.

A comprehensive list of commercial ICOs can be found on the website of www.bestcoins.co. Typically, a project tries to solve a problem within an industry and has associated coins with it. For example, the project Cargocoins tries to make the global shipping industry easier by a new crypto currency and act as an interactive way of sending, receiving, approving, rejecting and signing documents throughout the whole shipping process. By doing so, it reduces costs (e.g., banking fees), reduces fraud, minimizes delays and archives the whole process.

Within the banking sector the most widely used blockchain is called Ripple which started in 2012 to create a streamlined, decentralized payments system that tries to challenge the current SWIFT system (Leising and Robinson, 2018). Ripple has nothing to do with cryptocurrencies, it is just introducing a blockchain based system to transferring money around the world.

The future may give rise to Big Data Analytics (BDA) in combination blockchains for prescriptive analytics in the banking sector by combining data stemming from various sources (balance sheets, newspaper articles, reports on director's dealings in shares, chart analysis, macroeconomic data, exchange rates, TV interviews etc.) and blockchains to deliver customizable intelligent banking services to a client and vice versa i.e. the trustworthiness of an information can be proven by tracing it back to the source via a blockchain (Grishikashvili and Bechter, 2018).

Some observers argue that blockchains will replace bankers, lawyers, accountants and many other jobs. Smart contracts can help to exchange just anything. A simple example of a smart contract is the payment of the toll at a motorway (highway). Money gets deducted from the RFID card and the barrier opens. This happens in an automatic way without any negotiation or human interference. As soon as the conditions have been met (pay money) the results shows up (gate opens).

Figure 3 shows the research framework. A fiat cryptocurrency is a cryptocurrency which is issued by a central bank and therefore legal tender. It does not exist yet. Therefore, our research is looking into its feasibility. 


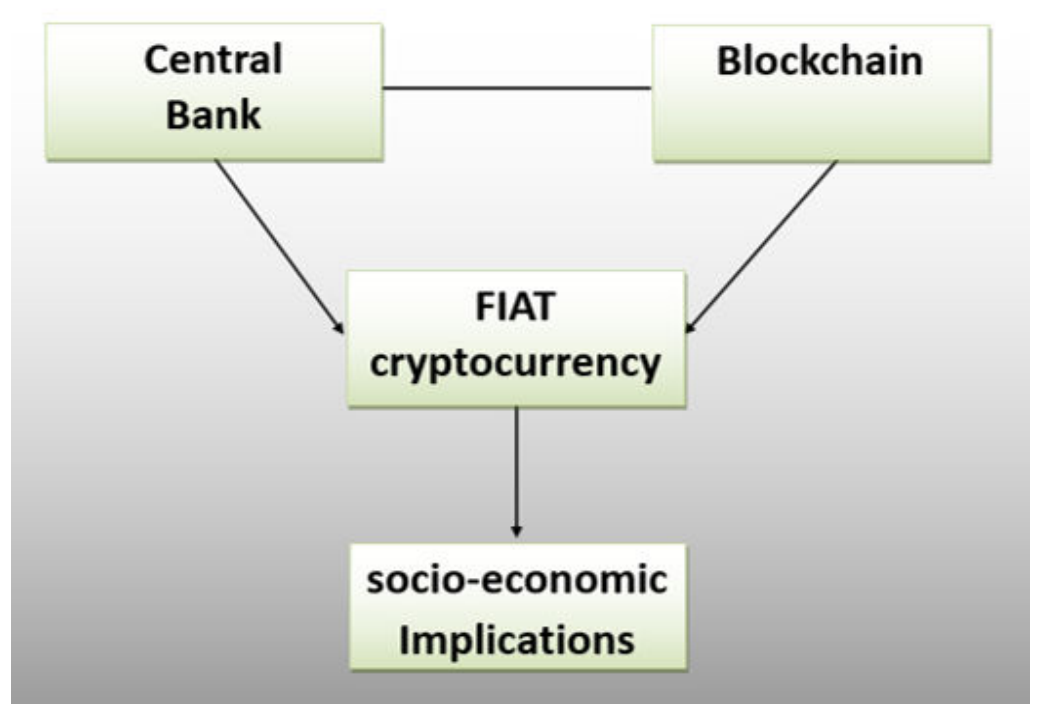

Figure 3. Research Framework

Most central banks are not enthused by the thought of cryptocurrencies and see rather the potential risks than the opportunities. In contrast, the European Central Bank is looking into the potential of blockchain technology to make financial markets more efficient and secure (Bott and Milkau, 2017).

\section{Vision for a Fiat Cryptocurrency}

Almost all countries have their own fiat (declared by a government to be legal tender) currency. Countries that do not have their own currency include: Andorra, Ecuador, East Timor, El Salvador, Marshall Islands, Micronesia, Palau, Palestine, Turks and Caicos, British Virgin Islands, Zimbabwe, and the Vatican. With one exception, all these countries chose voluntarily to adopting another currency despite they could issue their own. This exception is Palestine. This weakness could be turned into a strength and result in leapfrogging all advanced economies by being the first to introduce a cryptocurrency as legal tender. Palestine has a Monetary Authority (PMA) which comes close to a central bank. PMA is an independent public institution responsible for the formulation and implementation of monetary and banking policies and has maneuvered the country through difficult times (Riyad, 2016). Over its history, Palestine has used the Egyptian pound, then its own Palestinian Pound (equivalent to the UK Pound Sterling), then in 1948 accepted the Israeli Lira, then the Shekel and since 1985 the New Israeli Shekel (NIS). Today, Palestine does not have its own currency. In their daily lives Palestinians are using the NIS, the Euro, the US Dollar, and the Jordanian Dinar to conduct business. The PMA has little leverage to control money supply. Notoriously, Palestinian companies lack liquidity. However, research conducted in another Islamic country, Malaysia, suggests that there is no relationship between liquidity and profitability (Salam, 2016) which is a positive sign.

If the PMA decides to issue their own cryptocurrency, the new Palestinian Pound (PP), it will 


\section{Macrothink}

be backed by the central bank and will essentially become an official currency. There will be not much difference in comparison to a standard fiat currency, except that it will be blockchain based. PMA will be able to even print notes and coins if they want to and these can contain a QR code to be used, although that would probably not make much sense.

If PMA creates its own blockchain it could be developed to function exactly as any other fiat currency, i.e. initially PMA will have the full supply of coins that it is going to be distributed through banks and people will be able to exchange them for other currencies, such as the NIS or US\$.

The private blockchain platform, based on Hyperledger, could be modified to work in a way PMA wants, for example, it could be programmed to issue a specific amount of coins each year to account for inflation. To give trust in the PP currency, this inflation process could be capped to a maximum level of maybe $3 \%$ per year, so the PMA will not be tempted to issue more than 3\% new coins each year, otherwise it might cause inflation. The exchange rate of the PP against foreign currencies will be based on the supply/demand for PP coins. The price of the PP will fluctuate just like any other nation's currency. It will not fluctuate like a common cryptocurrency like Bitcoin. Since it will be built on a separate blockchain it will not be traded on crypto exchanges (e.g. coinbase.com), where a lot of speculations happen. It will be traded in the traditional way, just like any other nation's currency. Since it will be a convertible currency that anybody can buy from their bank at the exchange rate set by the central bank there will be no room for speculations and guarantee a stable currency which is important for an economy.

If PMA decides to launch the PP cryptocurrency, Palestine will have a chance to become the world's first nation with an official cryptocurrency, which will certainly attract a lot of positive global attention.

PMA could even do an ICO for a few million dollars and sell part of their PP tokens during an ICO. For example 50\% could go to Palestinians and the other $50 \%$ to private investors through an ICO. PMA could use a part of this money to build a fast IT infrastructure. 


\section{ETH raised}

\section{ETH 7,000}

ETH 14,000

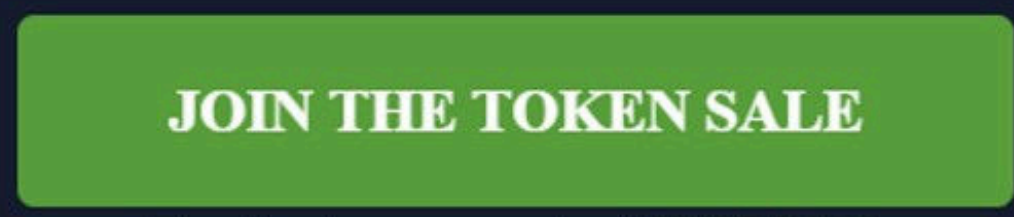

Total tokens supply: 100000000

Hard Cap: 14000 ETH

ICO Date: June 01 to July 15

Token Price: 1 ETH = 5,600 BITS Token

Currencies accepted: BTC, ETH

Figure 4. ICO Token Sale example

An ICO owner has to keep the investors well informed about the fundraising status, see Figure 4. Investors can usually enjoy a pre-sale discount in the region of $20 \%$. In above case an investor will receive 5,600 BITS Token (BitStump will become an exchange offering trading between popular currencies like the US\$ and cryptocurrencies) for one ETH. In this case one BITS coin would cost around 0.1 US\$. Most common is an ICO offering of one coin equaling one US\$.

In an ICO investors have to be convinced to buy into the PP idea. Investors want to know how the funds will be utilized (admin, marketing, R\&D etc.). In addition, they want to see a roadmap (timeline) for the coming four to five years and the team behind the project. The team includes advisors and public figures to give the ICO a trusted image. For spreading the word about the ICO a reward program called Bounty should be in place. One can for example write an article on the ICO or post a YouTube video or write a blog or any other promotional activity and the promotor will receive tokens as a reward. Even the translation of a whitepaper into another language will earn tokens.

Since it will be the first ICO done by a country and will be backed by a government, PMA could make the PP a huge success story. Estonia and India are currently preparing their own ICO to be launched with their own government issued cryptocurrency. If Palestine does it 
first, it can win the race and cash in on the first mover bonus. The only obstacle is the Paris Agreement of 1994 which forbids Palestine to have its own currency. However, there is widespread agreement that it is about time to move on and allow Palestinians to have their own currency (Koldas, 2017).

Other than using the cryptocurrency as means of exchange (i.e. money) Palestine could decide to do a full crypto approach, whereas the government could base all its operations on blockchain technology. If it does that, it could become the world's first and most advanced nation, which will attract a lot of attention and investment into the country. Blockchain could be used not just for payments, it could create a whole e-government based on blockchain and run public tenders, government documents, ID documents, credit/debit cards, and the banking system on it. The government could even do taxation through blockchain, so the taxation will be automatic, without the need for people or businesses to do anything or to submit anything. In the end the government could easily tax everybody without even having a tax authority set up and without having any tax inspectors, while at the same time having $100 \%$ tax collection at zero cost.

If Palestine decides to go for a full blockchain solution for its government, it could create a whole new political system. Although not being aware of blockchains at his time, Ronfeldt (1992) would call this a cyberocracy where a society makes highly effective use of information. Cyberocracy is the complete opposite to a traditional bureaucratic system with delays, corruption and inefficiency. Cyberocracy will be based on freedom and transparency, with minimal corruption level, while at the same time being much more efficient and faster than any other government system in the world.

The PP currency will have good and stable value in international markets, because all operations will be publicly visible and there will be no room for manipulations and printing of money. Forex traders will be completely aware of the real situation at any given moment in time, which will give predictability and full information disclosure on the currency front. Technically, prior to the launch, a Hackathon could be organized to look at options of how to implement the PP in detail.

\section{Conclusions}

What has been said about the PP applies to any other fiat cryptocurrency. If, for example, the Vatican decides to launch Vatican Coins then this will have similar implications.

Cryptocurrencies rely on blockchain technology. As such they are vulnerable to private key theft and malicious P2P attacks.

Our article looked at the possibility for the PP from a socio-economic perspective and purposely excluded political aspects which may be regarded as a limitation of our research.

Scope for further research will be the assessment of perceptions of citizens and the expected acceptance of the PP by national and international organizations. 


\section{References}

Berg, C. (2017). What Diplomacy in the Ancient Near East Can Tell Us About Blockchain Technology. Ledger, 2, 55-64. https://doi.org/10.5195/LEDGER.2017.104

Bott, J., \& Milkau, U. (2017). Central bank money and blockchain: A payments perspective. Journal of Payments Strategy \& Systems, 11(2), 145-157.

Corbet, S., Meegan, A., Larkin, C., Lucey, B., \& Yarovaya, L. (2018). Exploring the dynamic relationships between cryptocurrencies and other financial assets. Economics Letters, 16(5), 28-34. https://doi.org/10.1016/j.econlet.2018.01.004

Davies, S. (2015). How bitcoin and its blockchain work. Retrieved from https://www.ft.com/video/2be94381-66dc-3320-a292-6a1cde0a3d5f, accessed: 7.7.2018

Floyd, D. (2018). Chile Is Using Ethereum's Blockchain to Track Energy Data. Retrieved from https://www.coindesk.com/chile-to-use-ethereums-blockchain-to-track-energy-data/, accessed 7.7.2018

Garrett, R. (2017). How Blockchain is transforming the Supply Chain. Supply \& Demand Chain Executive, 18(2), 10-14.

Grishikashvili, K., \& Bechter, C. (2018). Perceptions of independent financial advisors on the usefulness of Big Data in the context of decision making in the UK. International Journal of Big Data Intelligence, 5(3), 1-12.

Heller, N. (2017). Its government is virtual, borderless, blockchained, and secure. Has this tiny post-Soviet nation found the way of the future? Retrieved from https://www.newyorker.com/magazine/2017/12/18/estonia-the-digital-republic, accessed 7.7.2018

Koldas, U. (2017). From Coercion to Consent?: The Paris Protocol and Contours of Hegemonic Economic Communication between the Israeli and Palestinian Societies. International Journal of Economic Perspectives, 11(1), 740-752.

Leising, M., \& Robinson, E. (2018). All Eyes on Ripple. But What Is It?. Bloomberg Businessweek, 4556, 29-31.

Meyer, D. (2018). Mining a Bitcoin Costs About as Much as Buying One These Days. Fortune.Com, 6.

Nation, J. (2018). Canada Leverages Ethereum Blockchain for Public Transparency of Government Grants. Retrieved from https://www.ethnews.com/canada-leverages-ethereum-blockchain-for-public-transparency-ofgovernment-grant, accessed 7.7.2018

Paynter, B. (2017). A new food chain. Fast Company, 219, 38-40.

PR, N. (2017, July 17). Grid+ Releases Whitepaper on Ethereum and Our Energy System. PR Newswire US.

Riyad, M. M. A. S. (2016). Business Continuity \& Disaster Recovery: Lessons from Palestine. International Journal of Management, 7(1), 26-41.

Ronfeldt, D. (1992). Cyberocracy is coming. The Information Society, 8(4), 243-296. https://doi.org/10.1080/01972243.1992.9960123 


\section{Macrothink}

Saeed, M. S., \& Zahid, N. (2016). The Impact of Credit Risk on Profitability of the Commercial Banks. Journal of Business \& Financial Affairs, 5, 192.

Salam, Z. A., Quoquab, F., Jamil, R., Rizal, A. M., Mahadi, N., \& Hussin, N. (2016). Advanced Science Letters, 22(12), 4506-4508. https://doi.org/10.1166/asl.2016.8204

SmartDubai. (2018). Dubai Blockchain Strategy, Retrieved from https://smartdubai.ae/en/Initiatives/Pages/DubaiBlockchainStrategy.aspx, accessed 7.7.2018

Weaver, N. (2018). Risks of Cryptocurrencies: Considering the inherent risks of cryptocurrency ecosystems. Communications of the ACM, 61(6), 20-24. https://doi.org/10.1145/3208095

Wei, W. C. (2018). Liquidity and market efficiency in cryptocurrencies. Economics Letters, 16(8), 21-24. https://doi.org/10.1016/j.econlet.2018.04.003

Young, J. (2018). Brazilian Government Plans to Process Petitions and Write Laws on Ethereum. Retrieved from https://cointelegraph.com/news/brazilian-government-plans-to-process-petitions-and-write-la ws-on-ethereum, accessed 7.7.2018

\section{Glossary}

BTC: Bitcoin

DAO: decentralized autonomous organizations

ETH: the coin Ethereum or short: Ether

ICO: initial coin offering

kb: kilobyte

NIS: New Israeli Shekel

PMA: Palestine Monetary Authority

P2P: peer-to-peer network

PP: cryptocurrency Palestinian Pound

QR code: two-dimensional barcode

UN: United Nations

\section{Copyright Disclaimer}

Copyright for this article is retained by the author(s), with first publication rights granted to the journal.

This is an open-access article distributed under the terms and conditions of the Creative Commons Attribution license (http://creativecommons.org/licenses/by/4.0/). 